\title{
Parental presence at induction of anesthesia is feasible with minimal preparation and resources
}

\author{
Phil Kruger, MBChB $\mathbb{D} \cdot$ David Rosen, MD
}

Received: 13 June 2016/Revised: 24 June 2016/Accepted: 14 July 2016/Published online: 4 August 2016

(C) Canadian Anesthesiologists' Society 2016

\section{To the Editor,}

Parental presence at the induction of anesthesia (PPI) in children has been practiced for decades. ${ }^{1,2}$ In response to families' requests, our centre set up a PPI program. A multidisciplinary working group was formed with the goal of implementing PPI without adversely affecting patient flow or increasing operating costs.

At our centre, volunteers accompany parents to and from the operating room (OR) and help explain the process. The volunteers have two days of orientation and come from all backgrounds. Parents receive an information pamphlet explaining PPI before their child's elective surgery. We sought to obtain families' opinions about the current PPI program as part of a quality improvement initiative after our institutional ethics board gave its approval for the program (approval number 20140141). Members of the multidisciplinary perioperative team developed a questionnaire. It was trialed for ease of use, acceptability, and accuracy. It was developed in English and translated into French.

In all, 284 questionnaires (see Appendix, available as Electronic Supplementary Material) were distributed to families coming for day surgery over a two-month period. Among them, 145 (51\%) were completed (111 in English, 41 in French). Patients' ages were 1-16 yr, with a mean (SD) age of 7.4 (4.0) yr. On a scale of 1-10, the overall satisfaction score of the PPI program was 9.3.

Electronic supplementary material The online version of this article (doi:10.1007/s12630-016-0707-x) contains supplementary material, which is available to authorized users.

P. Kruger, MBChB $(\bowtie) \cdot$ D. Rosen, MD

Children's Hospital of Eastern Ontario, Ottawa, ON, Canada

e-mail: pkruger@cheo.on.ca
Overall, $95 \%$ of parents indicated that they wanted to participate in PPI, and $87 \%$ were able to accompany their children. For those who did not, $3 \%$ of parents reported feeling that they did not need to, $3 \%$ reported that their child decided to go to the OR alone, and $7 \%$ had their participation negated by the anesthesiologist. In all, $96 \%$ of parents said that they would take part in PPI again, and $89 \%$ reported that their children expressed the desire for PPI in the future. Of the children who could express their opinion, $88 \%$ reported that it was helpful for them to have their parents accompany them. Of parents who participated in PPI, 99\% reported feeling well prepared for the process, although $43 \%$ of them were having their first surgical experience.

Approximately half of the patients received premedication with midazolam, and $86 \%$ of the parents reported that they thought it had helped. For the children who did not receive midazolam, $19 \%$ of parents said that they would request it next time.

The hospital's safety reporting system was queried regarding any potential adverse events related to parental behaviour or well-being associated with PPI; there were no reported events.

This survey was developed to provide information about the satisfaction of our hospital's families with our PPI program. The results indicated that families feel well prepared for PPI and are satisfied with the program. Many models for preparing families for PPI have been described, including written material, videos, and tours of the OR (both live and virtual). ${ }^{3}{ }^{5}$ We were encouraged by our results, given the small amount of preparation the families received before participating in PPI. We credit some of this success to the attentiveness of our volunteers, nurses, and child life specialists in the preoperative area. The comfort and familiarity of the 
OR staff, surgeons, and anesthesiologists with PPI also had an positive impact.

Anesthesiologists may refuse PPI because of concerns about patients' welfare. Specific examples include the need for rapid-sequence induction, patient fragility, or the complexity of the situation. Infants are excluded for the same reasons.

The study did have some limitations. The response rate of $51 \%$ likely would have been higher had we put a mechanism in place to prompt families to return completed forms. Additionally, families are likely to report high satisfaction scores with uncomplicated day surgery, which may have introduced bias into our results.

Parental presence at the induction of anesthesia in children has become an important part of our perioperative care, which is valued by our patients and their families. It allows families to "be there" for their children during a stressful time. It may also promote a deeper understanding of pediatric anesthesia. We have shown that a successful PPI program can be created with minimal additional cost and minimal resources, and that extensive parental preparation is not required. We speculate that it may be appropriate to expand this program to include patients of all ages, which may particularly benefit the elderly patient.
Disclosures None.

Competing interest None declared.

CHEO Research Ethics Board REB Protocol No: 14/59X. ROMEO File No: 20140141. Project Title: CHEOREB\#14/59X Family satisfaction on surgery day

Editorial responsibility This submission was handled by Dr. Philip M. Jones, Associate Editor, Canadian Journal of Anesthesia.

\section{References}

1. Scully SM. Parental presence during pediatric anesthesia induction. AORN J 2012; 96: 26-33.

2. Yip P, Middleton P, Cyna AM, Carlyle AV. Non-pharmacological interventions for assisting the induction of anaesthesia in children. Cochrane Database Syst Rev 2009; 3: CD006447.

3. Capurso M, Ragni B. Psycho-educational preparation of children for anaesthesia: a review of intervention methods. Patient Educ Couns 2016; 99: 173-85.

4. Bailey KM, Bird SJ, McGrath PJ, Chorney JE. Preparing parents to be present for their child's anesthesia induction: a randomized controlled trial. Anesth Analg 2015; 121: 1001-10.

5. Fortier MA, Kain $Z N$. Treating perioperative anxiety and pain in children: a tailored and innovative approach. Pediatr Anesth 2015; 25: 27-35. 